\title{
Prophylactic Ligation of the Median Cubital Vein to Improve the Patency of a Radio Cephalic Fistula
}

\author{
Chandrasekhar Chevuturu ${ }^{1, *}$, Mudigonda Somasekhar ${ }^{2}$, Sanjay Maitra $^{2}$, Manoj. K. Agarwala ${ }^{3}$ \\ ${ }^{1}$ Department of Vascular Surgery, Apollo Hospitals, Jubilee Hills, Hyderabad, India \\ ${ }^{2}$ Department of Nephrology, Apollo Hospitals, Jubilee Hills, Hyderabad, India \\ ${ }^{3}$ Department of Cardiology, Apollo Hospitals, Jubilee Hills, Hyderabad, India
}

Email address:

chandrasekhar1728@hotmail.com (C. Chevuturu)

\section{To cite this article:}

Chandrasekhar Chevuturu, Mudigonda Somasekhar, Sanjay Maitra, Manoj. K. Agarwala. Prophylactic Ligation of the Median Cubital Vein to Improve the Patency of a Radio Cephalic Fistula. Advances in Surgical Sciences. Vol. 4, No. 1, 2016, pp. 1-5.

doi: $10.11648 /$ j.ass.20160401.11

\begin{abstract}
The study evaluated the prophylactic ligation of the median cubital vein to improve the patency of a radiocephalic fistula in end stage renal disease (ESRD) patients undergoing hemodialysis. The study included a total of 38 ESRD patients undergoing arteriovenous fistula (AVF) surgery. Functional outcomes of the radiocephalic fistula were studied for six months by measuring primary patency rates. Out of 38 patients, 28 patients were cannulated at 20 days because of good flow and early maturation of the fistula. Revision AVF was performed on 4 patients after 3 months because of improper cannulation resulting in thrombosis of the cephalic vein. One patient underwent balloon angioplasty to maintain patency. Five patients discontinued the study. The primary patency rates for ESRD patients undergoing hemodialysis with radio cephalic fistula were better after ligation of the median cubital vein.
\end{abstract}

Keywords: Radio Cephalic Fistula, Median Cubital Vein, Ligation, Renal Disease

\section{Introduction}

Patients with end stage renal disease (ESRD) undergoing hemodialysis need good vascular access either through arteriovenous (AV) fistula, grafts or through central venous catheter [1]. AV fistula is the preferred method because of its higher longevity achieved by relatively lower rates of infection, thrombosis, septicemia, stenosis and complications $[2,3]$. AV accesses provide higher blood flow rates which further helps patients undergo effective dialysis [1].

Among various locations in the body, radio cephalic, brachio cephalic and brachio basilic regions are the best locations for successful creation of AV fistula. In patients with small caliber veins, direct creation of the fistulas can result in primary or secondary failures of the fistulas. Radiocephalic fistulas are created with the radial artery anastomosed to the cephalic vein. Various techniques have been described to prevent the failure of the fistula, which include single staged or two staged basilic vein transposition (BVT), single staged or two staged cephalic vein transposition (CVT), modified proximal radial artery arteriovenous fistula (MPRAVF), and a brachio axillary synthetic graft. A graft is usually an expensive option needing more intervention, and most of the above procedures are tedious and time consuming [2-8].

Dialysis access is a challenge for surgeons and for lifelong hemodialysis, patients maintaining adequate access usually requires more than one procedure. Chronic kidney disease patients have to undergo battery of blood tests and antibiotics from the cephalic vein which is the most superficial and easily cannulated vein. But most of the blood flow will be transferred from the fistula to uncannulated high caliber basilic vein and it results in poor maturation of cephalic vein in the arm [9-11]. Hence prophylactic ligation of median cubital vein was chosen.

Radiocephalic fistulas (RCF) make access easy and are used for dialysis due to a low complication rate [12, 13]. RCF performed by consultant surgeons in a study show primary and secondary patency rates of $80 \%$ and $93 \%$ at 22 months [14]. A new protocol for RCF creation involving ultrasound vein mapping and ligation of cephalic vein branches in the lower forearm had $82 \%$ functional patency rates [15]. The radio-median cubital vein/radiocephalic arterovenous fistula (RCAVF) at elbow is observed to be safe 
with better vascular access procedure for hemodialysis than brachiocephalic fistula [16]. The MPRAVF can be used to improve the success and patency of BVT and CVT [7].

Patients with AV fistula undergo dialysis after maturation which is assessed mainly by the dialysis staff. AV fistula will take on an average of 4-6 weeks to mature [17]. A flow of $>500 \mathrm{~mL} / \mathrm{min}$ is considered essential to prevent the thrombosis and blocking of the fistula [8, 18, 19]. Small caliber cephalic veins $(3-5 \mathrm{~mm})$ often act as a limiting factor for creation of a successful AVF at the forearm. In this study we have done the median cubital vein ligation through a separate incision in addition to the creation of a RCAVF. There were no animal studies tested on basilic vein ligation. The study evaluated prophylactic ligation of median cubital vein in dialysis patients in order to increase the blood flow to the cephalic vein and thus improve the patency of RCAVF.

\section{Methods}

A prospective study of cases undergoing surgery for venous access through the creation of AV fistula, were studied during a period of one year from 2011 to 2012. The inclusion criteria were ESRD patients undergoing hemodialysis with venous caliber of $3 \mathrm{~mm}$ and more. Total number of 38 patients having a venous caliber of $3 \mathrm{~mm}$ or more was included in this study. All the 38 patients were tested for patency by passing a 3 or 4 number fogarty's catheter up, to the level of the axilla to check the patency of cephalic vein during procedure. Patients with diabetes and or hypertension were noted.

The procedure included an additional incision given $2 \mathrm{~cm}$ below the ante cubital fossa to ligate the median cubital vein and other tributaries (Figure 2). A radio cephalic fistula was created at wrist level as per the standard procedure by performing an end to side anastomosis of the cephalic vein to radial artery (Figure 1). Follow up of all the patients undergoing the procedure for a period of 6 months was done to determine the functional outcomes of the radio cephalic fistula. Primary patency rates were used to evaluate the success of radio cephalic fistula.

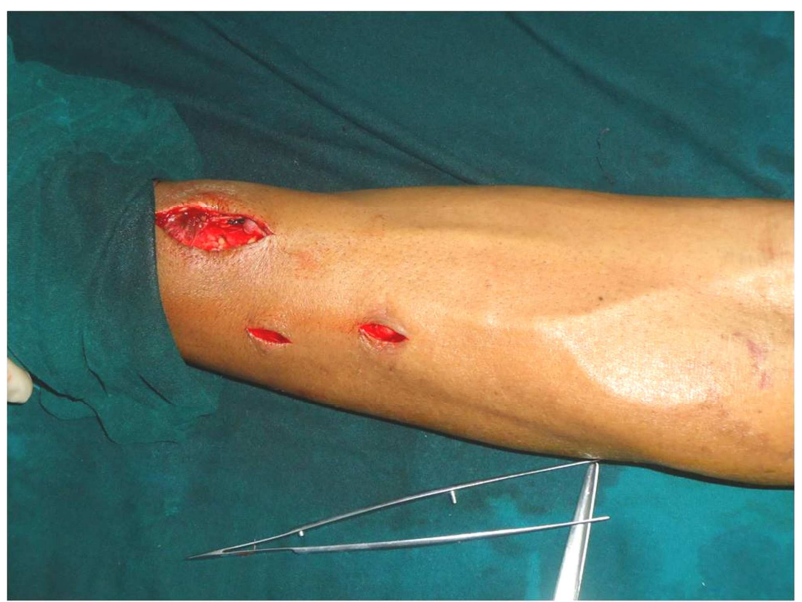

Figure 1. Photograph showing the procedure for creating AV Fistula.

\section{Results}

Out of 38 patients who underwent radio cephalic AV fistula creation at forearm, 5 patients lost follow-up; they were undergoing dialysis at another centre, and hence they have been excluded from the analysis. Out of the remaining 33 patients, the mean (SD) age was $52(2.5)$ years. There were four patients who dropped-out from the study. There were only 28patients who could be cannulated at 20 days. Although the correlation between the co-morbidities and functional outcomes was not done in the present study, $90.5 \%$ patients had hypertension, $87.4 \%$ had diabetes and $78 \%$ had both (Table 1$)$.

Table 1. Patient Demographics.

\begin{tabular}{ll}
\hline Base line characteristics & Number of patients/\% of patients \\
\hline Mean age, years & $52 \pm 2.5$ \\
Total Patients (n) & 33 \\
Patient dropouts (n) & 4 \\
Patients cannulated at 20 days (n) & 28 \\
Patients with hypertension (\%) & 90.5 \\
Patients with diabetes (\%) & 87.4 \\
$\begin{array}{l}\text { Patients with hypertension and } \\
\text { diabetes (\%) }\end{array}$ & 78 \\
\hline
\end{tabular}

Out of the 33 patients, 28 patients could be cannulated at 20 days because of good flow and early maturation of the fistula. Revision of AVF was performed on four patients after 3 months because of improper cannulation resulting in thrombosis of the cephalic vein at fore arm which was corrected by doing a proximal AVF at upper arm. One patient underwent balloon angioplasty to maintain patency (Table 2).

Table 2. Primary Patency Rates (6 months follow up).

\begin{tabular}{ll}
\hline Patients Primary Patency rates & Patients \\
\hline Total number of patients(excluding those who lost follow up) & 33 \\
Number of patients with good flow immediate post operation & 33 \\
Number of patients with flow at 6 months without intervention & 28 \\
Number of patients needing revision surgery & 4 \\
Number of patients needing angioplasty & 1 \\
\hline
\end{tabular}

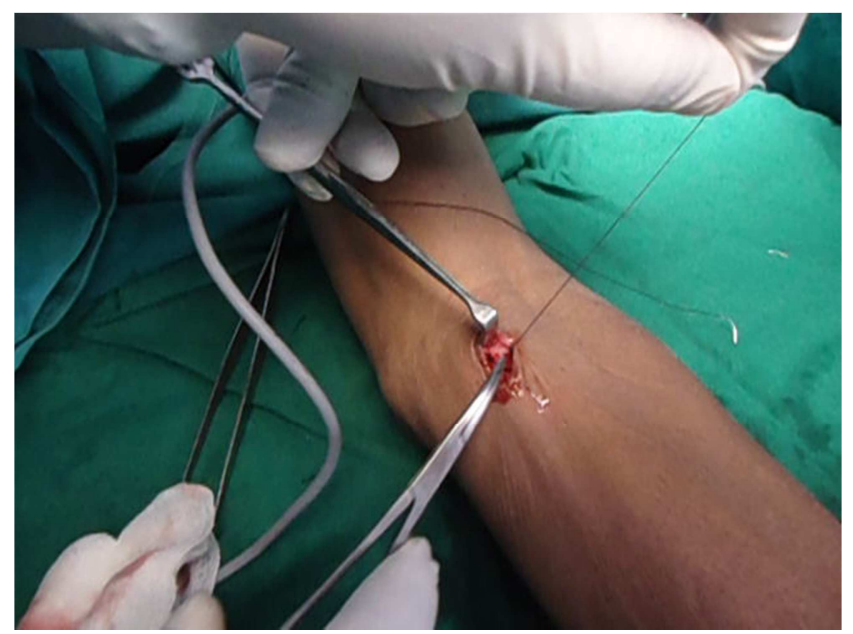

Figure 2. Photograph showing the procedure for ligation of medial cubital vein. 
During the six months of follow-up period, complications like edema of the corresponding arm, block, infections due to the procedure or failure of the procedure were not observed in any patient (Figure 3).

\section{Discussion}

Vascular access through AV fistula is the most commonly performed procedure for ESRD patients on hemodialysis [2-6]. There are two major considerations in selecting the type of the venous access. The first consideration is the maturation of the access to cannulate reproducibly and achieve a flow sufficient to provide adequate dialysis. Once maturation has occurred, the second consideration is to maintain long term patency of the access with minimal percutaneous or surgical intervention.

The major types of upper arm venous access include the AV graft, radio cephalic, brachio cephalic and transposed basilic vein. Though no particular data is available about the superiority of one location over the other, surgery at the level of the fore arm (radio cephalic) was chosen, in case the surgery at the wrist is not feasible due to narrow vessels. In case of failure of the fistula a new fistula can be created at the level of the elbow (brachio cephalic fistula).

The maturation time for a venous access through AVF is 4-6 weeks [18] and includes thickening of the vein, dilation of the vein and increase in diameter of the vein. The process takes even longer in the patients with small caliber veins as the flow is diverted into multiple tributaries (Figure 4). So vascular surgeons tend to perform a proximal brachio cephalic fistula to avoid failures by creating a radio cephalic fistula of poor caliber of $3 \mathrm{~mm}$ cephalic vein. Physical observation of the patients who underwent successful RCAVF in this study showed increase in the size of the vein (Figure 5).

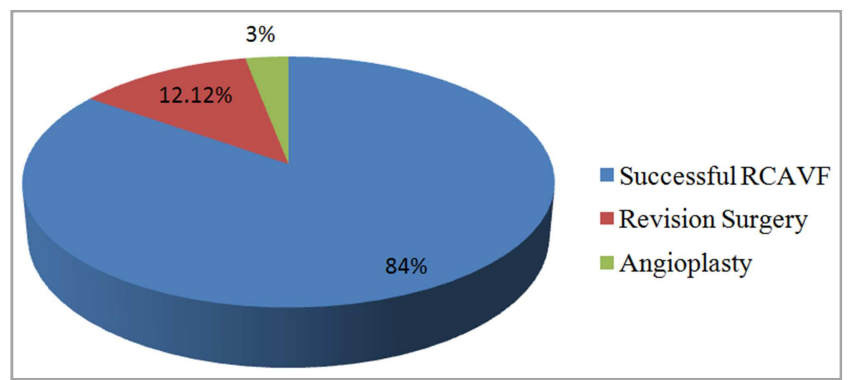

Figure 3. Primary Patency Distribution in Percentages (6 months follow up).

In AVF, as the high capacitance vein and the high velocity artery are connected, the blood flow through the AVF induces an increased shear wall stress. An increase in shear wall stress causes the endothelial release of nitric oxide which in turn increases the lumen radius for both acute and chronic shear wall alterations [20]. AVF cause an immediate 10-fold increase in blood flow and a three-fold increase in wall shear stress. Similar phenomenon of increase in blood flow was felt in the physical observation of patients who underwent successful RCAVF in this study (Figure 5) [21, 22]. The vessel enlargement starts within the first 24 hours. Lumen radius enlarges twofold at the end of 4 weeks and wall shear stress returns to normal. In this study visual observation showed increase in lumen radius in 3 weeks (Figure 5).

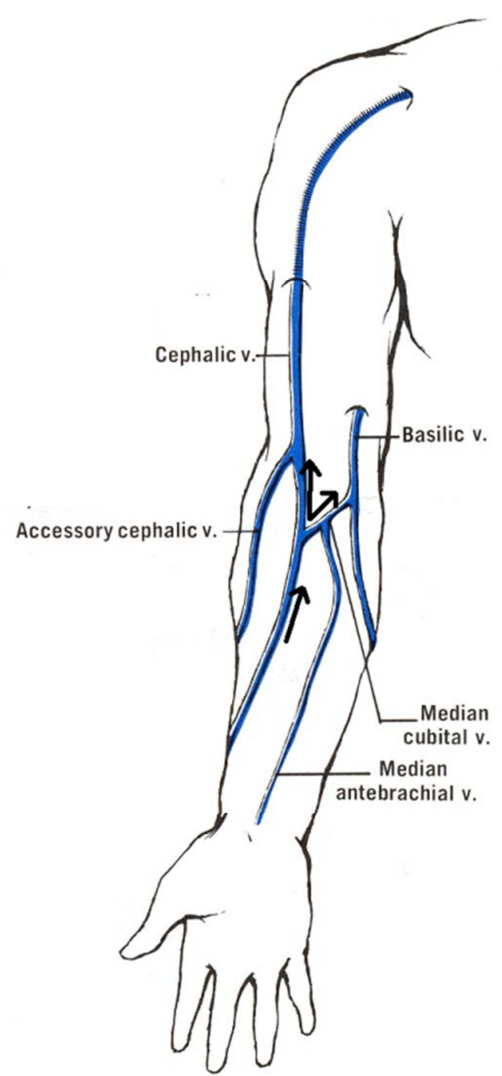

Figure 4. Diagram showing the flow of blood through a fistulised cephalic vein. Blood flows through both cephalic vein and basilic vein.

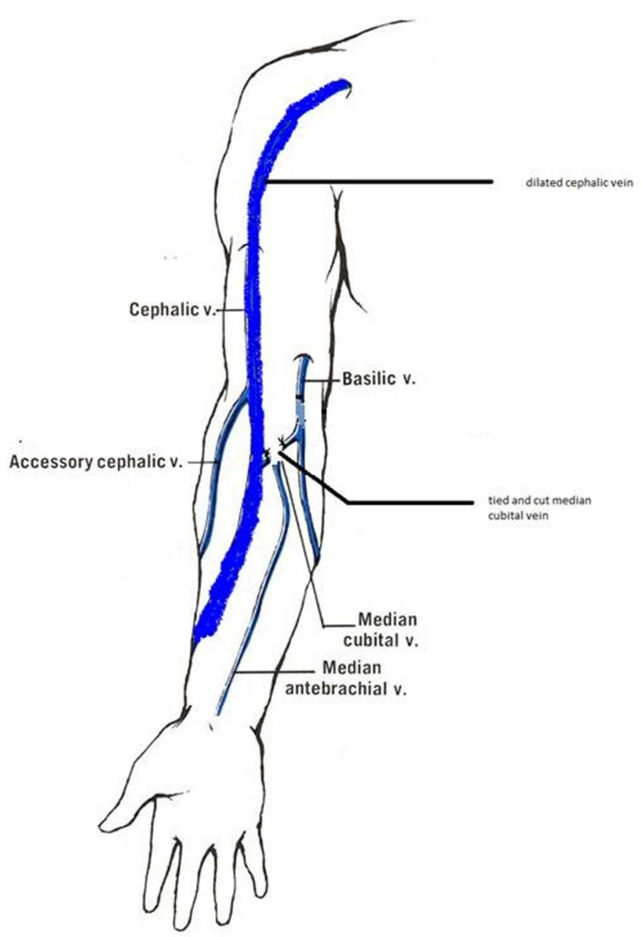

Figure 5. Diagram showing the effect of ligation of the median cubital vein showing the dilation. 
For the veins to be accessed for hemodialysis, the veins involved in the fistula creation should be superficial which include cephalic vein and the basilic vein. The basilic vein is the deeper vein and may drain the flow from the cephalic vein in the case an AV fistula created at the level of the fore arm. The idea behind ligating the median cubital vein is to restore and augment the flow of blood to cephalic vein helping in early maturation with better patency rates.

There are no long term consequences of ligation in dialysis patients and question of burning the potential bridge of access does not exist because proximal to ligated segment, basilic vein just collapses and will be drained by tributaries. Basilic vein never get thrombosed and may be useful for future basilic vein transposition.

As noted in our study, we did the ligation of the median cubital vein procedure in 38 patients with caliber less than
$3 \mathrm{~mm}$ out of which 33 were followed up and we had primary patency rates of $84 \%$. Complications and infections due to the procedure or failure of the procedure were not seen during the period of six months. Table 3 shows the comparison of radio cephalic fistula and their patency rates from various published studies.

Though the initial results with this technique seem to be encouraging, a longer follow up with more number of patients would be needed to establish better results in terms of primary patency, ease of access and maturation time. Diameter, flow volume measurements and dilatation of whole segment of cephalic vein of wrist level to upper arm can be noticed visually and cannulated time can be shortened. In this paper we report that this technique has given good results in ESRD patients undergoing hemodialysis with small caliber veins $(<3 \mathrm{~mm})$.

Table 3. Literature review summary of Radio Cephalic Fistulas Comparisons.

\begin{tabular}{lllll}
\hline S. No. & Reference & Number of patients studied & Technique used & Patency rates \\
\hline 1 & Burt et al.[23] & 53 & Radio cephalic fistula & At 2 years 60\% (>60yrs) 53\% (<60yrs) \\
2 & Fassiadis et al.[14] & 153 & Radio cephalic & patency $80 \%$ \\
3 & Nguyen et al.[24] & 114 & Radio cephalic & $50 \%$ \\
4 & Rooijens et al.[25] & Meta analysis of 38 studies & Radio cephalic fistula & $62.5 \%$ \\
5 & Present study & 38 & Radio cephalic fistula with basilic vein ligation & $94 \%$ \\
\hline
\end{tabular}

\section{Conclusions}

The results of the present study indicate that prophylactic ligation of the median cubital vein in radio cephalic fistula surgery yielded good results. The primary patency rates for ESRD patients undergoing hemodialysis with radio cephalic fistula were better after ligation of the median cubital vein. This technique is shown viable and should be evaluated in trials with more number of patients.

\section{Acknowledgements}

Author acknowledges the participants without whom this study would not have been accomplished.

\section{References}

[1] Manns B, Tonelli M, Yilmaz S, et al. Establishment and maintenance of vascular access in incident hemodialysis patients: a prospective cost analysis. J Am Soc Nephrol. 2005; 16(1): 201-209.

[2] Polkinghorne KR, McDonald SP, Atkins RC, et al. Vascular access and all-cause mortality: A propensity score analysis. $J$ Am Soc Nephrol. 2004; 15(2): 477-486.

[3] Astor BC, Eustace JA, Powe NR, et al. Type of vascular access and survival among incident hemodialysis patients: The Choices for Healthy Outcomes in Caring for ESRD (CHOICE) Study. J Am Soc Nephrol. 2005; 16(5): 1449-1455.

[4] Churchill DN, Taylor DW, Cook RJ, et al. Canadian Hemodialysis Morbidity Study. Am J Kidney Dis. 1992; 19(3): 214-234.
[5] Oliver MJ, McCann RL, Indridason OS, et al. Comparison of transposed brachiobasilic fistulas to upper arm grafts and brachiocephalic fistulas. Kidney Int. 2001; 60(4): 1532-1539.

[6] Dhingra RK, Young EW, Hulbert-Shearon TE, et al. Type of vascular access and mortality in US hemodialysis patients. Kidney Int. 2001; 60(4): 1443-1451.

[7] Hashemi H, Sheridan MJ, Ford B. Selective two-stage basilica and cephalic vein transpositions can significantly improve the rate of fistula construction. Int J Angiol. 2009; 18(3): 129-134.

[8] Tonelli M, Jindal K, Hirsch D, et al. Screening for subclinical stenosis in native vessel arteriovenous fistulae. $J$ Am Soc Nephrol. 2001; 12(8): 1729-1733.

[9] Casey K, Tonnessen BH, Mannava K, et al. Brachial versus basilic vein dialysis fistulas: a comparison of maturation and patency rates. J Vasc Surg. 2008; 47(2): 402-406.

[10] Martinez BD, LeSar CJ, Fogarty TJ, et al. Transposition of the basilic vein for arteriovenous fistula: an endoscopic approach. J Am Coll Surg. 2001; 192(2): 233-236.

[11] El Sayed HF, Mendoza B, Meier GH, et al. Utility of basilic vein transposition for dialysis access. Vascular. 2005; 13(5): 268-1674.

[12] Kim MH, Kim YK, Jun KW, Hwang JK, Kim SD, Kim JY, Park SC, Kim YS, Moon IS, Kim JI. Clinical Importance of Intraoperative Cephalic Vein Distensibility as a Predictor of Radiocephalic Arteriovenous Fistula Maturation. Semin Dial. 28(6): E64-70.

[13] Kordzadeh A, Chung J, Panayiotopoulos YP. Cephalic vein and radial arterydiameter in formation of radiocephalic arteriovenous fistula: a systematic review. $J$ Vasc Access. 2015; 16(6): 506-11.

[14] Fassiadis N, Morsy M, Siva M, et al. Does the surgeon's experience impact on radiocephalic fistula patency rates? Semin Dial. 2007; 20(5): 455-457. 
[15] Huseynova K, Werneck C, Pope M. A single center study of clinical success in radiocephalic fistula creation. $J$ Vasc Access. 2012; 13(2): 175-179.

[16] Kumar A, Jha MS, Singla M, et al. Radio-median cubital / radiocephalic arteriovenous fistula at elbow to prevent vascular steal syndrome associated with brachiocephalic fistula: Review of 320 cases. Indian J Urol. 2007; 23(3): 261164.

[17] Asif A, Roy-Chaudhury P, Beathard GA. Early arteriovenous fistula failure: a logical proposal for when and how to intervene. Clin J Am Soc Nephrol. 2006; 1(2): 332-339.

[18] Tonelli M, Hirsch D, Clark TW, et al. Access flow monitoring of patients with native vessel arteriovenous fistulae and previous angioplasty. J Am Soc Nephrol. 2002; 13(12): 29692973.

[19] Tonelli M, Jhangri GS, Hirsch DJ, et al. Best threshold for diagnosis of stenosis or thrombosis within six months of access flow measurement in arteriovenous fistulae. $\mathrm{J} \mathrm{Am} \mathrm{Soc}$ Nephrol. 2003; 14(12): 3264-3269.

[20] Sho E, Nanjo H, Sho M, et al. Arterial enlargement, tortuosity, and intimal thickening in response to sequential exposure to high and low wall shear stress. J Vasc Surg. 2004; 39(3): 601612 .

[21] Masuda H, Bassiouny H, Glagov S, et al. Artery wall restructuring in response to increased flow. Surg Forum. 1989; 45: 285-286.

[22] Hobson RW $2^{\text {nd }}$, Croom RD $3^{\text {rd }}$, Swan KG. Hemodynamics of the distal arteriovenous fistula in venous reconstruction. $J$ Surg Res. 1973; 14(5): 483-489.

[23] Burt CG, Little JA, Mosquera DA. The effect of age on radiocephalic fistula patency. $J$ Vasc Access. 2001; 2(3): 110113.

[24] Nguyen TH, Bui TD, Gordon IL, et al. Functional patency of autogenous AV fistulas for hemodialysis. $J$ Vasc Access. 2007; 8(4): 275-280.

[25] Rooijens PP, Tordoir JH, Stijnen T, et al. Radiocephalic wrist arteriovenous fistula for hemodialysis: meta-analysis indicates a high primary failure rate. Eur J Vasc Endovasc Surg. 2004; 28(6): 583-589. 